\title{
Analysis of Lossy Transmission Lines Terminated by Schottky Diode Circuit
}

\author{
Vasil G. Angelov
}

\begin{abstract}
In the present paper we consider a lossy transmission line terminated by a circuit corresponding to a Schottky diode. On the base of Kirchhoff's law boundary conditions are derived. Then a mixed problem for the lossy transmission line system is formulated. We reduce the mixed problem for the hyperbolic transmission line system to an initial value problem for a system of differential equations with delays on the boundary. We prove existence-uniqueness theorem for oscillatory solution. The paper ends with numerical example with real values of the Schottky diode parameters.
\end{abstract}

Index Terms-Fixed Point Method; Lossy Transmission Line; Oscillatory Solution; Schottky Diode.

\section{INTRODUCTION}

The main purpose of the present paper is to investigate the propagation of signals along a lossy transmission line terminated by a circuit corresponding to a Schottky-barrier diode. In this case the circuit consists of parallel connected nonlinear $R C$-loads and in series connected $R$-load (cf. [1] [4]). The nonlinear characteristics of $R C$-loads generate nonlinearities in the differential equations on the boundary with delays. Our considerations are under the Heaviside condition $L / R=G / C$, where $L, R, G, C$ are specific parameters of the transmission line ( $L$ is per unit-length inductance, $R$ - per unit-length resistance, $G$ - per unitlength conductance, $C$ - per unit-length capacitance) and $\Lambda$ is its length, $v=1 / \sqrt{L C}$ ) is the speed of propagation and $T=\Lambda /(1 / \sqrt{L C})=\Lambda \sqrt{L C}$ is the time delay.

The paper consists of seven sections. First we formulate the mixed problem for the hyperbolic system describing a lossy transmission line and derive the boundary conditions (on the base of the Kirchhoff's law) generated by Schottky diode circuit. We emphasize that in this case the $R C$ current function cannot be eliminated but we extend our method (cf. [5]-[8]) and consider three equations introducing additional current (voltage). Various applications of such circuits are given in [9] - [24]. In Section 2 we transform the hyperbolic system in a diagonal form. In Section 3 we reduce the mixed problem to an initial value problem on the boundary. We show that natural solutions are oscillatory ones. In Section 4 we analyze nonlinearities and give an operator presentation of the oscillatory problem. In other words, we introduce an operator whose fixed point is an oscillatory solution of the

Published on September 15, 2018

V. G. Angelov is with the Department of Mathematics, Faculty of Mining and Electro-mechanics, University of Mining and Geology "St. I. Rilski”, 1700 Sofia, Bulgaria (e-mail: angelov@mgu.bg). initial value problem on the boundary. Section 5 contains a preliminary assertion. In Section 6 we prove the main result - the existence-uniqueness theorem of an oscillatory solution of differential system with delays. Finally, in Section 7, we give a numerical example which demonstrates the advantages of our method.

Here we consider a lossy transmission line described by the system

$$
\begin{aligned}
& \frac{\partial u(x, t)}{\partial x}+L \frac{\partial i(x, t)}{\partial t}+R i(x, t)=0 \\
& \frac{\partial i(x, t)}{\partial x}+C \frac{\partial u(x, t)}{\partial t}+G u(x, t)=0
\end{aligned}
$$

which can be rewritten in the matrix form

$$
\frac{\partial U(x, t)}{\partial t}+A \frac{\partial U(x, t)}{\partial x}+A_{1} U=\overline{0}
$$

where

$$
\begin{gathered}
U=\left[\begin{array}{c}
u(x, t) \\
i(x, t)
\end{array}\right], \frac{\partial U}{\partial t}=\left[\begin{array}{c}
\frac{\partial u(x, t)}{\partial t} \\
\frac{\partial i(x, t)}{\partial t}
\end{array}\right], \frac{\partial U}{\partial x}=\left[\begin{array}{c}
\frac{\partial u(x, t)}{\partial x} \\
\frac{\partial i(x, t)}{\partial x}
\end{array}\right], \\
A=\left[\begin{array}{cc}
0 & 1 / C \\
1 / L & 0
\end{array}\right], A_{1}=\left[\begin{array}{cc}
G / C & 0 \\
0 & R / L
\end{array}\right] .
\end{gathered}
$$

Formulate mixed problem for (1): to find a solution $u(x, t), i(x, t)$ for $(x, t) \in \Pi=\left\{(x, t) \in R^{2}: 0 \leq x \leq \Lambda, t \geq 0\right\}$, satisfying the initial conditions

$u(x, 0)=u_{0}(x), i(x, 0)=i_{0}(x)$ for $x \in[0, \Lambda]$

and boundary conditions derived bellow.

The Schottky barrier diode has an equivalent circuit shown on Fig. 1. Here $C_{0}$ and $R_{1}$ are nonlinear capacitance and resistance, while $R_{2}$ - linear resistance.

For the capacitance we choose (cf. Maas [1])

$$
Q_{0}(u)=-C_{j 0} \Phi(1-(u / \Phi))^{1-\gamma} /(1-\gamma)
$$

and consequently $C_{0}(u)=\frac{d Q_{0}(u)}{d u}=C_{j 0}(1-(u / \Phi))^{-\gamma}$, where constants $C_{j 0}, \Phi, \gamma$ are explained finally in the numerical example.

The current of the capacitance is

$$
i_{C_{0}}=\frac{d Q_{0}\left(u_{C_{0}}\right)}{d u} \frac{d u_{C_{0}}}{d t}=C_{j 0}\left(1-\left(u_{C_{0}} / \Phi\right)\right)^{-\gamma} \frac{d u_{C_{0}}}{d t} .
$$




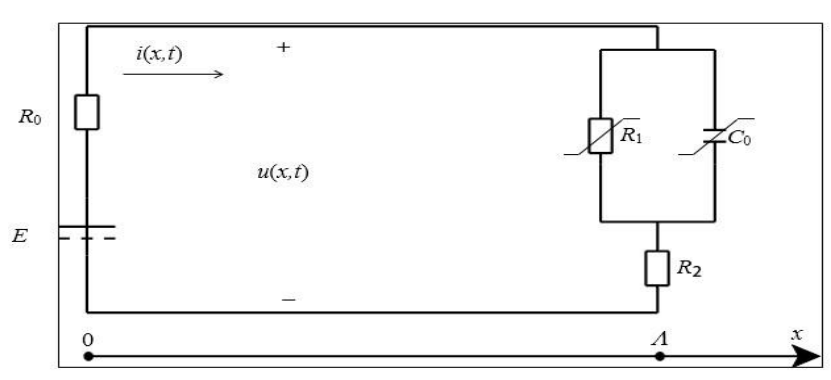

Fig. 1. Lossy transmission line loaded by Schottky diode circuit

If $\left|u_{C_{0}}\right| \leq \phi_{0} e^{\mu_{0}}<\Phi$ then $d C_{0}(u) / d u$ has strictly positive lower bounds and upper bound. Indeed,

$$
\frac{d C_{0}\left(u_{C_{0}}\right)}{d u_{C_{0}}}=C_{j 0} \gamma\left(1-\left(u_{C_{0}} / \Phi\right)\right)^{-\gamma-1} / \Phi>0
$$

and therefore

$$
C_{j 0}\left(1+\left(\phi_{0} e^{\mu_{0}} / \Phi\right)\right)^{-\gamma} \leq C_{0}\left(u_{C_{0}}\right) \leq C_{j 0}\left(1-\left(\phi_{0} e^{\mu_{0}} / \Phi\right)\right)^{-\gamma} .
$$

The current of the resistance $R_{1}$ is

$$
i_{R_{1}}\left(u_{R_{1}}\right)=I_{\text {sat }}\left(e^{\alpha u R_{1}}-1\right) \text {. }
$$

Obviously both elements have nonlinear characteristics that generate similar nonlinearities in the boundary conditions.

Indeed, applying the Kirchhoff's law for the right end of the line we have $i_{C_{0}}+i_{R_{1}}=i(\Lambda, t)$. But $u_{C_{0}}=u_{R_{1}}$ and $u_{R_{1}}+u_{R_{2}}=u(\Lambda, t) \Leftrightarrow u_{C_{0}}+u_{R_{2}}=u(\Lambda, t)$. Therefore

$$
\begin{gathered}
C_{j 0}(1-(u / \Phi))^{-\gamma} \frac{d u_{C_{0}}}{d t}+I_{s a t}\left(e^{\alpha u} C_{0}-1\right)=i(\Lambda, t) ; \\
u_{C_{0}}+R_{2} i_{R_{2}}=u(\Lambda, t) .
\end{gathered}
$$

But $i_{R_{2}}=i(x, t)$. In this manner we obtain the boundary conditions (cf. Fig. 1) for $x=0$

$$
E(t)-u(0, t)-R_{0} i(0, t)=0, t \geq 0
$$

and in view of $i_{R_{2}}(\Lambda, t)=i(\Lambda, t)$ for $x=\Lambda$

$$
\begin{aligned}
& u_{C_{0}}+R_{2} i(\Lambda, t)=u(\Lambda, t), \\
& C_{j 0}(1-(u / \Phi))^{-\gamma} \frac{d u_{C_{0}}}{d t}+I_{s a t}\left(e^{\alpha{ }^{u} C_{0}}-1\right)=i(\Lambda, t)
\end{aligned}
$$

\section{TRANSFORMATION OF THE HYPERBOLIC SYSTEM IN A DIAGONAL FORM}

Consider the system (2). The eigenvalues of the matrix $A$ are $\lambda_{1}=1 / \sqrt{L C}, \lambda_{2}=-1 / \sqrt{L C}$ and corresponding eigenvectors are $\left(\xi_{1}^{(1)}, \xi_{2}^{(1)}\right)=(\sqrt{C}, \sqrt{L}),\left(\xi_{1}^{(2)}, \xi_{2}^{(2)}\right)=(-\sqrt{C}, \sqrt{L})$.

The matrix $H$ formed by the eigenvectors is $H=\left[\begin{array}{cc}\sqrt{C} & \sqrt{L} \\ -\sqrt{C} & \sqrt{L}\end{array}\right]$ and its inverse is $H^{-1}=\left[\begin{array}{rr}1 /(2 \sqrt{C}) & -1 /(2 \sqrt{C}) \\ 1 /(2 \sqrt{L}) & 1 /(2 \sqrt{L})\end{array}\right]$.

It is known that $A^{\text {can }}=H A H^{-1}$, where

$$
A^{\mathrm{can}}=\left[\begin{array}{ll}
1 / \sqrt{L C} & 0 \\
0 & -1 / \sqrt{L C}
\end{array}\right] \text {. }
$$

Introduce new variables $Z=\left[\begin{array}{c}V(x, t) \\ I(x, t)\end{array}\right]$, where $Z=H U$ and $U=H^{-1} Z$ or

$$
\mid \begin{aligned}
& V(x, t)=\sqrt{C} u(x, t)+\sqrt{L} i(x, t) \\
& I(x, t)=-\sqrt{C} u(x, t)+\sqrt{L} i(x, t)
\end{aligned}
$$

and

$$
\mid \begin{aligned}
& u(x, t)=(V(x, t)-I(x, t)) /(2 \sqrt{C}) \\
& i(x, t)=(V(x, t)+I(x, t)) /(2 \sqrt{L}) .
\end{aligned}
$$

Substituting $U=H^{-1} Z$ in (2) we obtain

$$
\begin{gathered}
\frac{\partial\left(H^{-1} Z\right)}{\partial t}+A \frac{\partial\left(H^{-1} Z\right)}{\partial x}+A_{1}\left(H^{-1} Z\right)=\overline{0}, \\
H^{-1} \frac{\partial Z}{\partial t}+A H^{-1} \frac{\partial Z}{\partial x}+A_{1}\left(H^{-1} Z\right)=\overline{0} .
\end{gathered}
$$

Then multiplying the last matrix equality by $H$ from the left we obtain

$$
\frac{\partial Z}{\partial t}+H A H^{-1} \frac{\partial Z}{\partial x}+\left(H A_{1} H^{-1}\right) Z=\overline{0}
$$

where $H A_{1} H^{-1}=\frac{1}{2}\left[\begin{array}{cc}G / C+R / L & -G / C+R / L \\ -G / C+R / L & G / C+R / L\end{array}\right]$.

We assume that Heaviside condition $G / C=R / L$ is fulfilled. Then $H A_{1} H^{-1}=\left[\begin{array}{cc}R / L & 0 \\ 0 & R / L\end{array}\right]$ and the hyperbolic system becomes

$$
\frac{\partial V}{\partial t}+\frac{1}{\sqrt{L C}} \frac{\partial V}{\partial x}+\frac{R}{L} V=0, \frac{\partial I}{\partial t}-\frac{1}{\sqrt{L C}} \frac{\partial I}{\partial x}+\frac{R}{L} I=0 .
$$

Let us put $V(x, t)=e^{-R t / L} W(x, t), \quad I(x, t)=e^{-R t / L} J(x, t)$.

Substituting in the last system we reach $\frac{\partial W(x, t)}{\partial t}+\frac{1}{\sqrt{L C}} \frac{\partial W(x, t)}{\partial x}=0, \frac{\partial J(x, t)}{\partial t}-\frac{1}{\sqrt{L C}} \frac{\partial J(x, t)}{\partial x}=0$.

Finally, the transformation formulas are

$$
\mid \begin{aligned}
& W(x, t)=e^{R t / L} \sqrt{C} u(x, t)+e^{R t / L} \sqrt{L} i(x, t) \\
& J(x, t)=-e^{R t / L} \sqrt{C} u(x, t)+e^{R t / L} \sqrt{L} i(x, t)
\end{aligned}
$$

and

$$
\mid \begin{aligned}
& u(x, t)=e^{-R t / L} W(x, t) /(2 \sqrt{C})-e^{-R t / L} J(x, t) /(2 \sqrt{C}) \\
& i(x, t)=e^{-R t / L} W(x, t) /(2 \sqrt{L})+e^{-R t / L} J(x, t) /(2 \sqrt{L}) .
\end{aligned}
$$

Prior to formulate an operator corresponding to the mixed problem we consider the Cauchy problems for the characteristics (Here we mean the characteristics of the hyperbolic system):

$$
\frac{d x_{W}(\tau)}{d \tau}=1 / \sqrt{L C}, x_{W}(t)=x ; \frac{d x_{J}(\tau)}{d \tau}=-1 / \sqrt{L C}, x_{J}(t)=x
$$

for each $(x, t) \in \Pi$. Here the characteristic functions $\lambda_{W}=1 / \sqrt{L C}>0$ and $\lambda_{J}=-1 / \sqrt{L C}<0$ are continuous ones.

We need the following

Lemma 1. For every $(x, t) \in$ int $\Pi$ the initial value 
problem

$$
\frac{d x_{W}(\tau)}{d \tau}=1 / \sqrt{L C}, x_{W}(t)=x ;\left(\frac{d x_{J}(\tau)}{d \tau}=-1 / \sqrt{L C}, x_{J}(t)=x\right)
$$

has a unique solution belonging to $\Pi$.

\section{Reducing the MiXed Problem to AN Initial VALUE PROBLEM ON THE BOUNDARY}

Here we formulate the mixed problem: to find a solution $W(x, t), J(x, t)$ satisfying the system

$\frac{\partial W(x, t)}{\partial t}+\frac{1}{\sqrt{L C}} \frac{\partial W(x, t)}{\partial x}=0, \frac{\partial J(x, t)}{\partial t}-\frac{1}{\sqrt{L C}} \frac{\partial J(x, t)}{\partial x}=0$,

initial conditions

$$
\mid \begin{aligned}
& W(x, 0)=\sqrt{C} u(x, 0)+\sqrt{L} i(x, 0)=\sqrt{C} u_{0}(x)+\sqrt{L} i_{0}(x) \equiv W_{0}(x) \\
& J(x, t)=-\sqrt{C} u(x, 0)+\sqrt{L} i(x, 0)=-\sqrt{C} u_{0}(x)+\sqrt{L} i_{0}(x) \equiv J_{0}(x)
\end{aligned},
$$

and boundary conditions for $x=0$ :

$E(t)-u(0, t)-R_{0} i(0, t)=0, t \geq 0$

and for $x=\Lambda$ :

$C_{j 0}(1-(u / \Phi))^{-\gamma} \frac{d u_{C_{0}}}{d t}+I_{s a t}\left(e^{\alpha u} C_{0}-1\right)=i(\Lambda, t)$;

$u_{C_{0}}(t)+R_{2} i_{R_{2}}(\Lambda, t)=u(\Lambda, t)$

which can be transformed in view of

$u(0, t)=e^{-R t / L}(W(0, t)-J(0, t)) /(2 \sqrt{C})$

$i(0, t)=e^{-R t / L}(W(0, t)+J(0, t)) /(2 \sqrt{L})$,

$u(\Lambda, t)=e^{-R t / L}(W(\Lambda, t)-J(\Lambda, t)) /(2 \sqrt{C})$

$i(\Lambda, t)=e^{-R t / L}(W(\Lambda, t)+J(\Lambda, t)) /(2 \sqrt{L})$

in the form

$$
\begin{aligned}
& E(t)-e^{-R t / L}(W(0, t)+J(0, t)) /(2 \sqrt{C}) \\
& -e^{-R t / L}\left(R_{0} W(0, t)-R J(0, t)\right) /(2 \sqrt{L})=0, t \geq 0 ; \\
& u_{C_{0}}+R_{2} e^{-R t / L}(W(\Lambda, t)+J(\Lambda, t)) /(2 \sqrt{L}) \\
& =e^{-R t / L}(W(\Lambda, t)-J(\Lambda, t)) /(2 \sqrt{C}) ; \\
& \quad C_{j 0} \quad \frac{d u_{C_{0}}(t)}{d t}+I_{s a t}\left(e^{\alpha u_{C_{0}}(t)}-1\right) \\
& =e^{-R t / L}(W(\Lambda, t)+J(\Lambda, t)) /(2 \sqrt{L}) .
\end{aligned}
$$

Integrating the first equation of (7) along the first characteristic we obtain

$$
\begin{aligned}
& \int_{t}^{t+T}\left(\frac{\partial W}{d x} \frac{d x_{W}}{d s}+\frac{\partial W}{\partial s}\right) d s=\int_{t}^{t+T} \frac{d W\left(x_{W}(s), s\right)}{d s} d s= \\
& =W(\Lambda, t+T)-W(0, t)=0 .
\end{aligned}
$$

In a similar way integrating along the second characteristic from $(0, t+T)$ to $(\Lambda, t)$ we obtain

$$
\int_{t+T}^{t}\left(\frac{\partial J}{d x} \frac{d x_{J}}{d s}+\frac{\partial J}{\partial s}\right) d s=\int_{t+T}^{t} \frac{d J\left(x_{J}(s), s\right)}{d s} d s=J(\Lambda, t)-J(0, t+T)=0
$$

Therefore $W(\Lambda, t)=W(0, t-T), J(\Lambda, t-T)=J(0, t)$.

We assume that $W(0, t)=W(t) ; J(\Lambda, t)=J(t)$ are unknown functions. Then the boundary conditions become

$$
\begin{aligned}
& E(t)-e^{-R t / L}(W(t)-J(t-T)) /(2 \sqrt{C}) \\
& -e^{-R t / L}\left(R_{0} W(t)+R J(t-T)\right) /(2 \sqrt{L})=0 ; \\
& u_{C_{0}}+e^{-R t / L} R_{2}(W(t-T)+J(t)) /(2 \sqrt{L}) \\
& =e^{-R t / L}(W(t-T)-J(t)) /(2 \sqrt{C}) ; \\
& C_{j 0}\left(1-\left(u_{C_{0}} / \Phi\right)\right)^{-\gamma} \frac{d u_{C_{0}}(t)}{d t}+I_{s a t}\left(e^{\alpha u^{u} C_{0}}-1\right) \\
& =e^{-R t / L}(W(t-T)+J(t)) /(2 \sqrt{L}) .
\end{aligned}
$$

It is proved in [8] that the above relations are equivalent to the mixed problem for the hyperbolic system. Using the denotation $Z_{0}=\sqrt{L / C}$ we present the last equations in a fixed point form and we are able to formulate the main problem: to find an oscillatory solution $\left(W(t), J(t), u_{C_{0}}(t)\right)$ of the system

$$
\begin{aligned}
& W(t)=\frac{2 e^{R t / L} E(t) \sqrt{L}}{Z_{0}+R_{0}}+\frac{Z_{0}-R_{0}}{Z_{0}+R_{0}} J(t-T), \\
& J(t)=-\frac{2 e^{R t / L} u_{C_{0}}(t) \sqrt{L}}{Z_{0}+R_{2}}+\frac{Z_{0}-R_{2}}{Z_{0}+R_{2}} W(t-T), \\
& \frac{d u_{C_{0}}(t)}{d t}=-\left(1-\left(u_{C_{0}}(t) / \Phi\right)\right)^{\gamma} / C_{j 0} \\
& \cdot\left(I_{s a t}\left(e^{\alpha u C_{0}}-1\right)-(W(t-T)+J(t)) e^{-R t / L} /(2 \sqrt{L})\right), \\
& W(t)=W_{0}(t), \quad J(t)=J_{0}(t), t \in[0, T]
\end{aligned}
$$

with prescribed initial functions on $[0, T]$ obtained from (8) as in [8]. The constants $\alpha, \Phi, \gamma, I_{\text {sat }}, C_{j 0}$ will be commented in the numerical example at the very end of the paper. The right-hand sides of (9) show that natural solutions are oscillatory ones. Periodic solutions do not exist in view of $e^{-R t / L}$

\section{OPERATOR PRESENTATION OF THE OSCILLATORY PROBLEM}

Here we introduce an operator acting on a uniform space of oscillatory functions [8]. Its fixed points are oscillatory solutions of the problem in question (9).

We look for a solution of (9) with advanced prescribed zeroes on $\left[t_{0}=T, \infty\right)$, First we define the sets of zeros. The prescribed initial functions $W_{0}(t), J_{0}(t), t \in[0, T]$ have zeros $\tau_{0}=0<\tau_{1}<\ldots<\tau_{n}=T$. Then we translate this picture on $[T, 2 T],[2 T, 3 T], \ldots$, that is,

$$
\begin{aligned}
& t_{0}=T<t_{1}=\tau_{1}+T<\ldots<t_{n}+T=2 T, \\
& 2 T=t_{n+1}=t_{n}+T<t_{n+2}=t_{n}+T \ldots<t_{n+n}+T=3 T, \ldots .
\end{aligned}
$$

It follows that $t_{k}-T=t_{k-n}$ for every $k$.

Denote by $T_{0}=\max \left\{\tau_{k+1}-\tau_{k}: k=0,1, \ldots, n-1\right\}$.

Obviously $S=\left\{t_{k}\right\}_{k=0}^{\infty}$ is a strictly increasing sequence 
for which $\lim _{k \rightarrow \infty} t_{k}=\infty$.

By $C\left[t_{0}, \infty\right)$ we denote the set of continuous and bounded functions on interval $\left[t_{0}, \infty\right)$. The initial functions $W_{0}(),. J_{0}(.) \in C[0, T]$ satisfy the inequalities:

$$
\left|W_{0}(t)\right| \leq W_{0} e^{\mu\left(t-\tau_{k}\right)},\left|J_{0}(t)\right| \leq J_{0} e^{\mu\left(t-\tau_{k}\right)}, t \in\left[\tau_{k}, \tau_{k+1}\right],
$$

$(k=0,1,2, \ldots, n-1)$.

Introduce the sets

$M_{W}=\left\{W(.) \in C\left[t_{0}, \infty\right): W\left(t_{k}\right)=0 \wedge|W(t)| \leq W_{0} e^{\mu\left(t-t_{k}\right)}\right\}$,

$M_{J}=\left\{J(.) \in C\left[t_{0}, \infty\right): J\left(t_{k}\right)=0 \wedge|J(t)| \leq J_{0} e^{\mu\left(t-t_{k}\right)}\right\}$,

$M_{0}=\left\{u_{C_{0}}(.) \in C\left[t_{0}, \infty\right): u_{C_{0}}\left(t_{k}\right)=0 \wedge\left|u_{C_{0}}(t)\right| \leq \phi_{0} e^{(\mu-(R / L))\left(t-t_{k}\right)}\right\}$

for $t \in\left[t_{k}, t_{k+1}\right]$.

Assumption $(\Phi): \phi_{0} e^{\mu T_{0}}<\Phi$.

Here $W_{0}, J_{0}, \phi_{0}, \mu, \mu T_{0}=\mu_{0}$ are positive constants.

Introduce the following family of pseudo-metrics

$\rho_{k}(V, \bar{V})=\max _{t \in\left[t_{k}, t_{k+1}\right]}\left\{e^{-\mu\left(t-t_{k}\right)}|V(t)-\bar{V}(t)|\right\}$,

$\rho_{k}(J, \bar{J})=\max _{t \in\left[t_{k}, t_{k+1}\right]}\left\{e^{-\mu\left(t-t_{k}\right)}|J(t)-\bar{J}(t)|\right\}$,

$\rho_{k}\left(u_{C_{0}}, \bar{u}_{C_{0}}\right)=\max _{t \in\left[t_{k}, t_{k+1}\right]}\left\{e^{-(\mu-(R / L))\left(t-t_{k}\right)}\left|u_{C_{0}}(t)-\bar{u}_{C_{0}}(t)\right|\right\}$.

The set $(X, A)=M_{W} \times M_{J} \times M_{0}$ turns out into a

complete uniform space (cf. [25]) with respect to the countable family of pseudo-metrics $(k=0,1,2, \ldots)$

$\rho_{k}\left(\left(W, J, u_{C_{0}}\right),\left(\bar{W}, \bar{J}, \bar{u}_{C_{0}}\right)\right)$

$=\max \left\{\rho_{k}(W, \bar{W}), \rho_{k}(J, \bar{J}), \rho_{k}\left(u_{C_{0}}, \bar{u}_{C_{0}}\right)\right\}$.

Here the index set is $A=\{0,1,2, \ldots\}$.

A map $B:(X, A) \rightarrow(X, A)$ is called $\Phi$ - contractive iff $\rho_{k}\left(B\left(W, J, u_{C_{0}}\right), B\left(\bar{W}, \bar{J}, \bar{u}_{C_{0}}\right)\right) \leq L_{0} \rho_{j(k)}\left(B\left(W, J, u_{C_{0}}\right), B\left(\bar{W}, \bar{J}, \bar{u}_{C_{0}}\right)\right)$ $(k=0,1,2, \ldots), L_{0}<1$, where the map $j: A \times X \times X \rightarrow A$ is defined as follows: $j^{0}(k)=k$,

$j(k)=$

$$
\left\{\begin{aligned}
k, \text { if } \max & \left\{\rho_{k-n}(W, \bar{W}), \rho_{k-n}(J, \bar{J}), \rho_{k}(J, \bar{J}), \rho_{k}\left(u_{C_{0}}, \bar{u}_{C_{0}}\right)\right\} \\
& \text { is attended for } k \\
k-n, \text { if } \max \left\{\rho_{k-n}(W, \bar{W}), \rho_{k-n}(J, \bar{J}), \rho_{k}(J, \bar{J}), \rho_{k}\left(u_{C_{0}}, \bar{u}_{C_{0}}\right)\right\} & \text { is attended for } k-n .
\end{aligned}\right.
$$

and $j^{m}(k)=j\left(j^{m-1}(k)\right)$. Obviously after every translation $t-T$ moves every interval $\left[t_{k}, t_{k+1}\right]$ to the left and after finite number of iterations its image turns out to the left of the initial point or does not depend on $m$. Consequently

$$
\rho_{j^{m}(k)}\left(\left(W, J, u_{C_{0}}\right),\left(\bar{W}, \bar{J}, \bar{u}_{C_{0}}\right)\right) \leq Q<\infty \quad(m=0,1,2,3 \ldots),
$$

that is, the uniform space is $j$-bounded (cf. [25], [26]). This implies a uniqueness of the fixed point of the $\Phi-$ contractive map.

Prior to define a operator $B$ we consider the space $C[0, \infty)$ with zeros $\left\{\tau_{k}\right\}_{k=0}^{n} \cup\left\{t_{k}\right\}_{k=0}^{\infty}$ which coincide with $W_{0}($.$) on [0, T]$. In view of the definition of $\left\{\tau_{k}\right\}_{k=0}^{n}$ $\cup\left\{t_{k}\right\}_{k=0}^{\infty}$ every translated to the right function (with length $T)$ coincides with some function $\tilde{W}(.) \in C\left[t_{0}, \infty\right)$. In a similar way we proceed for $\tilde{J}(.) \in C\left[t_{0}, \infty\right)$.

Now we are able to define the operator $B\left(W, J, u_{C_{0}}\right)=\left(B_{W}\left(W, J, u_{C_{0}}\right), B_{I}\left(W, J, u_{C_{0}}\right), B_{0}\left(W, J, u_{C_{0}}\right)\right):$ $M_{W} \times M_{J} \times M_{0} \rightarrow M_{W} \times M_{J} \times M_{0}$

by the formulas

$B_{W}\left(W, J, u_{C_{0}}\right):=\left\{\begin{array}{c}J_{0}(t), t \in[0, T] ; \\ \frac{2 e^{R t / L} E(t) \sqrt{L}}{Z_{0}+R_{0}}+\frac{Z_{0}-R_{0}}{Z_{0}+R_{0}} \tilde{J}(t), t \in[T, \infty)\end{array}\right.$
$B_{I}\left(W, J, u_{C_{0}}\right):=\left\{\begin{array}{c}W_{0}(t), t \in[0, T] \\ -\frac{2 e^{R t / L} u_{C_{0}}(t) \sqrt{L}}{Z_{0}+R_{2}}+\frac{Z_{0}-R_{2}}{Z_{0}+R_{2}} \tilde{W}(t), t \in[T, \infty)\end{array}\right.$,

$B_{0}\left(W, J, u_{C_{0}}\right):=\int_{t_{k}}^{t} U_{k}\left(W, J, u_{C_{0}}\right)(s) d s-\frac{t-t_{k}}{t_{k+1}-t_{k}} \int_{t_{k}}^{t_{k}+1} U_{k}\left(W, J, u_{C_{0}}\right)(s) d s$ $t \in\left[t_{k}, t_{k+1}\right],(k=0,1,2, \ldots)$,

where

$$
\begin{aligned}
& U_{k}\left(W, J, u_{C_{0}}\right)(t)=\left(1-\left(u_{C_{0}}(t) / \Phi\right)\right) \gamma /\left(C_{j_{0}}\right) \\
& \cdot\left[-I_{s a t}\left(e^{\alpha u} C_{0}{ }^{(t)}-1\right)+e^{-R\left(t-t_{k}\right) / L}(\tilde{W}(t)+J(t)) /(2 \sqrt{L})\right] .
\end{aligned}
$$

\section{PRELIMINARY ASSERTIONS}

Lemma 2. If $2-\left|\alpha u_{C_{0}}(t)\right| \geq 2-\alpha \phi_{0} e^{\mu_{0}}>0$, then (9) has a solution $\left(W, J, u_{C_{0}}\right) \in M_{W} \times M_{J} \times M_{0}$ iff the operator $B$ has a fixed point in $M_{W} \times M_{J} \times M_{0}$, that is,

$\left(W, J, u_{C_{0}}\right)=\left(B_{0}\left(B_{W}\left(W, J, u_{C_{0}}\right), B_{J}\left(W, J, u_{C_{0}}\right), B_{0}\left(W, J, u_{C_{0}}\right)\right)\right.$.

Proof: Let $\left(V, I, u_{C_{0}}\right) \in M_{V} \times M_{I} \times M_{0}$ be a solution of (9). For the first and second equation the assertion is obvious. Then integrating the third equation of (9) on the interval $\left[t_{k}, t\right] \subset\left[t_{k}, t_{k+1}\right](k=0,1,2 \ldots)$ we obtain

$u_{C_{0}}(t)-u_{C_{0}}\left(t_{k}\right)=\int_{t_{k}}^{t} U_{k}\left(W, J, u_{C_{0}}\right)(s) d s$

$\Leftrightarrow u_{C_{0}}(t)=\int_{t_{k}}^{t} U_{k}\left(W, J, u_{C_{0}}\right)(s) d s$.

To reach

$u_{C_{0}}(t)=\int_{t_{k}}^{t} U_{k}\left(W, J, u_{C_{0}}\right)(s) d s-\frac{t-t_{k}}{t_{k+1}-t_{k}} \int_{t_{k}}^{t_{k}+1} U_{k}\left(W, J, u_{C_{0}}\right)(s) d s$

we notice

$u_{C_{0}}(t)=\int_{t_{k}}^{t} U_{k}\left(W, J, u_{C_{0}}\right)(s) d s \Rightarrow 0=u_{C_{0}}\left(t_{k+1}\right)=\int_{t_{k}}^{t_{k+1}} U_{k}\left(W, J, u_{C_{0}}\right)(s) d s$.

Therefore the ordered triple $\left(W, J, u_{C_{0}}\right)$ satisfies the system

$W(t)=\left(2 e^{R t / L} E(t) \sqrt{L}+\left(Z_{0}-R_{0}\right) J(t-T)\right) /\left(Z_{0}+R_{0}\right)$, $J(t)=\left(-2 e^{R t / L} u_{C_{0}}(t) \sqrt{L}+\left(Z_{0}-R_{2}\right) W(t-T)\right) /\left(Z_{0}+R_{0}\right)$,

$u_{C_{0}}(t)=\int_{t_{k}}^{t} U_{k}\left(W, J, u_{C_{0}}\right)(s) d s-\frac{t-t_{k}}{t_{k+1}-t_{k}} \int_{t_{k}}^{t_{k+1}} U_{k}\left(W, J, u_{C_{0}}\right)(s) d s$

for $t \in\left[t_{k}, t_{k+1}\right]$, that is, $\left(W, J, u_{C_{0}}\right)$ is a fixed point of $B$. 
Conversely, let $\left(W, J, u_{C_{0}}\right)$ be a fixed point of $B$, that is, $\left(W, J, u_{C_{0}}\right) \quad$ satisfies $\quad(10) . \quad$ Then $\quad\left(\mu_{0}=\mu T_{0}=\mathrm{const}\right)$ assuming $\mu>R / L$ and introducing $E_{\mu_{0}}=e^{(\mu-(R / L)) T_{0}}$ we obtain

$$
\begin{aligned}
& \left|\int_{t_{k}}^{t_{k+1}} U_{k}\left(W, J, u_{C_{0}}\right)(s) d s\right| \leq\left(1 / C_{j_{0}}\right) \mid \int_{t_{k}}^{t_{k}+1}\left(1+\left|u_{C_{0}}(s)\right| / \Phi\right)^{\gamma}
\end{aligned}
$$

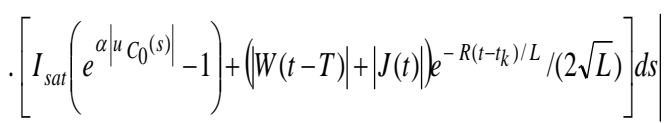

$$
\begin{aligned}
& \leq\left(1 / C_{j_{0}}\right) \mid \int_{t_{k}}^{t_{k}+1}\left(1+\phi_{0} e^{(\mu-(R / L))\left(t-t_{k}\right)} / \Phi\right)^{\gamma} \\
& {\left[I_{s a t}\left(e^{\alpha \phi_{0} e^{\left(\mu-(R / L)\left(t-t_{k}\right)\right.}}-1\right)+\left(W_{0} e^{-\mu T}+J_{0}\right) e^{(\mu-(R / L))\left(t-t_{k}\right)} /(2 \sqrt{L})\right] d t} \\
& \leq\left(1+\phi_{0} E_{\mu_{0}} / \Phi\right) / C_{j_{0}}
\end{aligned}
$$

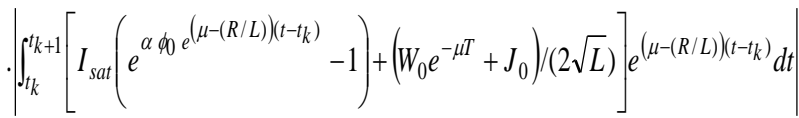

$$
\begin{aligned}
& \leq\left(1+\phi_{0} E_{\mu_{0}} / \Phi\right) y\left|\int_{t_{k}}^{t_{k+1}} e^{(\mu-(R / L))\left(t-t_{k}\right)} d t\right| / C_{j_{0}} \\
& \cdot\left[I_{\text {sat }}\left(e^{\alpha \phi_{0} e^{(\mu-(R / L))\left(t-t_{k}\right)}}-1\right)+\left(W_{0} e^{-\mu T}+J_{0}\right) /(2 \sqrt{L})\right] \\
& \leq\left(1+\phi_{0} E_{\mu_{0}} / \Phi\right) \gamma\left(e^{(\mu-(R / L))\left(t_{k+1}-t_{k}\right)}-1\right) / C_{j 0}(\mu-(R / L)) \\
& \cdot\left[I_{\text {sat }}\left(e^{\alpha \phi_{0} E_{\mu_{0}}}-1\right)+\left(W_{0} e^{-\mu T}+J_{0}\right) /(2 \sqrt{L})\right] \\
& \leq\left(1+\left(\phi_{0} E_{\mu_{0}} / \Phi\right)\right) r\left(E_{\mu_{0}}-1\right) / C_{j 0}(\mu-(R / L)) \\
& \cdot\left[I_{\text {sat }}\left(e^{\alpha \phi_{0} E_{0}}-1\right)+\left(W_{0} e^{-\mu T}+J_{0}\right) /(2 \sqrt{L})\right] \text {. }
\end{aligned}
$$

It follows $\int_{t_{k}}^{t_{k+1}} U_{k}\left(W, J, u_{C_{0}}\right)(s) d s=0$ because $\mu>0$ could be chosen sufficiently large. Therefore

$u_{C_{0}}(t)=\int_{t_{k}}^{t} U_{k}\left(W, J, u_{C_{0}}\right)(s) d s-\frac{t-t_{k}}{t_{k+1}-t_{k}} \int_{t_{k}}^{t_{k+1}} U_{k}\left(W, J, u_{C_{0}}\right)(s) d s$ $\Leftrightarrow u_{C_{0}}(t)=\int_{t_{k}}^{t} U_{k}\left(W, J, u_{C_{0}}\right)(s) d s$.

Differentiating the last integral equation we obtain the third equation of (9).

Lemma 2 is thus proved.

\section{EXISTENCE-UNIQUENESS OF AN OSCILLATORY SOLUTION}

Theorem 1. Let the following conditions be fulfilled:

1) $E(t):[0, \infty) \rightarrow(-\infty, \infty), \quad E\left(t_{k}\right)=0,|E(t)| \leq W_{0} e^{(\mu-(R / L))\left(t-t_{k}\right)}$, $t \in\left[t_{k}, t_{k+1}\right],(k=0,1,2, \ldots)$;

2) The initial functions $W_{0}(),. J_{0}(.) \in C[0, T]$ satisfy the conditions $W_{0}\left(\tau_{k}\right)=0, J_{0}\left(\tau_{k}\right)=0$ and

$\left|W_{0}(t)\right| \leq W_{0} e^{\mu\left(t-\tau_{k}\right)},\left|J_{0}(t)\right| \leq J_{0} e^{\mu\left(t-\tau_{k}\right)}, t \in\left[\tau_{k}, \tau_{k+1}\right]$,

$(k=0,1,2, \ldots, m-1)$;

3) $2-\alpha \phi_{0} e^{\mu T_{0}}>0,2 \phi_{0} \sqrt{C} \leq W_{0}$;

4) $\left(2 \sqrt{L} W_{0}+\left|Z_{0}-R_{0}\right|\right) J_{0} /\left(Z_{0}+R_{0}\right) \leq W_{0}$;
5) $\left(Z_{0}+\left|Z_{0}-R_{2}\right|\right) W_{0} /\left(Z_{0}+R_{2}\right) \leq J_{0}$;

6) $\left(1+\left(\phi_{0} E_{\mu_{0}} / \Phi\right)\right)^{\gamma} E_{\mu_{0}} / C_{j 0}(\mu-(R / L))$

$$
\text { . }\left[I_{s a t}\left(e^{\alpha \phi_{0} E_{\mu_{0}}}-1\right)+\left(W_{0}+J_{0}\right) /(2 \sqrt{L})\right] \leq \phi_{0} \text {; }
$$

7) $K_{J}=\left(2 \sqrt{L}+\left|Z_{0}-R_{2}\right|\right) /\left(Z_{0}+R_{2}\right)<1$;

8) $K_{C}=E_{\mu_{0}}\left(1+\left(\phi_{0} E_{\mu_{0}} / \Phi\right)\right) /\left(\mu C_{j 0}\right)$

$\cdot\left\{\left((\gamma / \Phi)\left(I_{s a t} e^{\alpha \phi_{0} E \mu_{0}}+\left(W_{0}+J_{0}\right) /(2 \sqrt{L})\right)\right)\right.$

$\left..\left(1-\left(\phi_{0} E_{\mu_{0}} / \Phi\right)\right)^{-1}\left(E_{\mu_{0}}-1\right) / 2+I_{s a t} e^{\alpha \phi_{0} E_{\mu_{0}}}+1 / \sqrt{L}\right\}<1$.

Then there exists a unique oscillatory solution of (9).

Proof: First we show that

$B=\left(B_{W}\left(W, J, u_{C_{0}}\right), B_{J}\left(W, J, u_{C_{0}}\right), B_{0}\left(W, J, u_{C_{0}}\right)\right)$ :

$M_{W} \times M_{J} \times M_{0} \rightarrow M_{W} \times M_{J} \times M_{0}$.

Indeed, it is easy to see that $B_{W}\left(W, J, u_{C_{0}}\right)(t)$ and

$B_{J}\left(W, J, u_{C_{0}}\right)(t)$ are oscillatory functions. Indeed,

$B_{0}^{(k-1)}\left(W, J, u_{C_{0}}\right)\left(t_{k}\right)$

$=\int_{t_{k-1}}^{t_{k}} U_{k-1}\left(W, J, u_{C_{0}}\right)(s) d s-\frac{t_{k}-t_{k-1}}{t_{k}-t_{k-1}} \int_{t_{k-1}}^{t_{k}} U_{k-1}\left(W, J, u_{C_{0}}\right)(s) d s=0$,

$B_{0}^{(k)}\left(W, J, u_{C_{0}}\right)\left(t_{k}\right)$

$=\int_{t_{k}}^{t_{k}} U_{k}\left(W, J, u_{C_{0}}\right)(s) d s-\frac{t_{k}-t_{k}}{t_{k+1}-t_{k}} \int_{t_{k}}^{t_{k+1}} U_{k}\left(W, J, u_{C_{0}}\right)(s) d s=0$.

To verify their continuity we notice

$W_{0}(T)=\frac{2 e^{R T / L} E(T) \sqrt{L}}{Z_{0}+R_{0}}+\frac{Z_{0}-R_{0}}{Z_{0}+R_{0}} J(0)$

$J_{0}(T)=-\frac{2 e^{R T / L} u_{C_{0}}(T) \sqrt{L}}{Z_{0}+R_{2}}+\frac{Z_{0}-R_{2}}{Z_{0}+R_{2}} W(0)$,

$B_{0}^{(k)}\left(W, J, u_{C_{0}}\right)\left(t_{k+1}\right)=\int_{t_{k}}^{t_{k}+1} U_{k}\left(W, J, u_{C_{0}}\right)(s) d s$

$-\frac{t_{k+1}-t_{k}}{t_{k+1}-t_{k}} \int_{t_{k}}^{t_{k+1}} U_{k}\left(W, J, u_{C_{0}}\right)(s) d s=0$,

$B_{0}^{(k+1)}\left(W, J, u_{C_{0}}\right)\left(t_{k+1}\right)=\int_{t_{k+1}}^{t_{k+1}} U_{k+1}\left(W, J, u_{C_{0}}\right)(s) d s$

$-\frac{t_{k+1}-t_{k+1}}{t_{k+2}-t_{k+1}} \int_{t_{k+1}}^{t_{k+2}} U_{k+1}\left(W, J, u_{C_{0}}\right)(s) d s=0$,

$t \in\left[t_{k}, t_{k+1}\right],(k=0,1,2, \ldots)$.

The following inequalities are valid for $t \in\left[t_{k}, t_{k+1}\right]$, $(k=0,1,2, \ldots)$ :

$\left|B_{W}\left(W, J, u_{C_{0}}\right)(t)\right|$

$\leq\left(2 e^{R t / L}|E(t)| \sqrt{L}+\left|Z_{0}-R_{0}\right||J(t-T)|\right) /\left(Z_{0}+R_{0}\right)$

$\leq\left(2 W_{0} e^{\mu\left(t-t_{k}\right)} \sqrt{L}+\left|Z_{0}-R_{0}\right| J_{0} e^{\mu\left(t-t_{k}\right)} e^{-\mu T}\right) /\left(Z_{0}+R_{0}\right)$

$\leq\left(2 W_{0} \sqrt{L}+\left|Z_{0}-R_{0}\right| J_{0}\right) e^{\mu\left(t-t_{k}\right)} /\left(Z_{0}+R_{0}\right) \leq W_{0} e^{\mu\left(t-t_{k}\right)}$

and

$\left|B_{J}\left(W, J, u_{C_{0}}\right)(t)\right|$

$\leq\left(2 e^{R t / L} u_{C_{0}}(t) \sqrt{L}+\left|Z_{0}-R_{2} \| W(t-T)\right|\right) /\left(Z_{0}+R_{2}\right)$

$\leq\left(2 \sqrt{L} \phi_{0}+\left|Z_{0}-R_{2}\right| W_{0} e^{-\mu T}\right) e^{\mu\left(t-t_{k}\right)} /\left(Z_{0}+R_{2}\right)$ 


$$
\leq\left(2 Z_{0} \sqrt{C} \phi_{0}+\left|Z_{0}-R_{2}\right| W_{0} e^{-\mu T}\right) e^{\mu\left(t-t_{k}\right)} /\left(Z_{0}+R_{2}\right)
$$$$
\leq e^{\mu\left(t-t_{k}\right)}\left(Z_{0}+\left|Z_{0}-R_{2}\right|\right) W_{0} /\left(Z_{0}+R_{2}\right) \leq J_{0} e^{\mu\left(t-t_{k}\right)} .
$$

Using (10) we obtain

$\left|B_{0}\left(W, J, u_{C_{0}}\right)(t)\right| \leq\left|\int_{t_{k}}^{t} U_{k}\left(W, J, u_{C_{0}}\right)(s) d s\right|+$

$+\left|\left(t-t_{k}\right) /\left(t_{k+1}-t_{k}\right)\right|\left|\int_{t_{k}}^{t_{k+1}} U_{k}\left(W, J, u_{C_{0}}\right)(s) d s\right| ;$

$\left.\left|\int_{t_{k}}^{t} U_{k}\left(W, J, u_{C_{0}}\right)(s) d s\right| \leq\left(1 / C_{j 0}\right) \mid \int_{t_{k}}^{t}\left(1+\left(\left|u_{C_{0}}(s)\right| / \Phi\right)\right)\right)^{\prime}$

$\cdot\left[I_{s a t}\left(e^{\alpha\left|{ } C_{0}(s)\right|}-1\right)+(|W(s-T)|+|J(s)|) e^{-R\left(s-t_{k}\right) / L} /(2 \sqrt{L})\right] d s \mid$

$\left.\leq\left(1 / C_{j 0}\right) \int_{t_{k}}^{t}\left(1+\phi_{0} e^{(\mu-(R / L))\left(s-t_{k}\right)} / \Phi\right)\right)^{y}$

$\cdot\left[I_{s a t}\left(e^{\alpha \phi_{0} e^{(\mu-(R / L))\left(s-t_{k}\right)}}-1\right)+\left(W_{0}+J_{0}\right) e^{(\mu-(R / L))\left(s-t_{k}\right)} /(2 \sqrt{L})\right] d s \mid$

$\leq\left(1+\phi_{0} e^{(\mu-(R / L)) T_{0}} / \Phi\right)^{\gamma /} / C_{j 0}$

|| $\int_{t_{k}}^{t}\left[I_{s a t}\left(e^{\alpha \phi_{0} E_{0}}-1\right)+\left(W_{0}+J_{0}\right) /(2 \sqrt{L})\right] e^{(\mu-(R / L))\left(s-t_{k}\right)} d s \mid$

$\leq\left[I_{\text {sat }}\left(e^{\alpha \phi_{0} E_{\mu}}-1\right)+\left(W_{0}+J_{0}\right) /(2 \sqrt{L})\right] / C_{j 0}$

$\left(1+\phi_{0} E_{\mu_{0}} / \Phi\right)\left|\int_{t_{k}}^{t} e^{(\mu-(R / L))\left(s-t_{k}\right)} d s\right|$

$\leq\left(1+\phi_{0} E_{\mu_{0}} / \Phi\right) \gamma\left(e^{(\mu-(R / L))\left(t-t_{k}\right)}-1\right) / C_{j 0}(\mu-(R / L))$

$\cdot\left[I_{\text {sat }}\left(e^{\alpha \phi_{0} E_{\mu_{0}}}-1\right)+\left(W_{0}+J_{0}\right) /(2 \sqrt{L})\right]$.

In a similar way

$\left|\frac{t-t_{k}}{t_{k+1}-t_{k}}\right|\left|\int_{t_{k}}^{t_{k+1}} U\left(W, J, u_{C_{0}}\right)(s) d s\right|$

$\leq\left(1+\phi_{0} E_{\mu_{0}} / \Phi\right)\left(E_{\mu_{0}}-1\right) /(\mu-(R / L))$

$\cdot\left[I_{\text {sat }}\left(e^{\alpha \phi_{0} E_{0}}-1\right)+\left(W_{0}+J_{0}\right) /(2 \sqrt{L})\right] / C_{j 0}$.

Therefore

$\left|B_{0}\left(W, J, u_{C_{0}}\right)(t)\right| \leq$

$\leq\left(1 / C_{j 0}\right)\left(1+\phi_{0} E_{\mu_{0}} / \Phi\right)^{k}$

. $\left[I_{\text {sat }}\left(e^{\alpha \phi_{0} E_{\mu_{0}}}-1\right)+\left(W_{0}+J_{0}\right) /(2 \sqrt{L})\right]$

. $\left(\left(e^{(\mu-(R / L))\left(t-t_{k}\right)}+\left(E_{\mu_{0}}-1\right) e^{(\mu-(R / L))\left(t-t_{k}\right)}\right) /(\mu-(R / L))\right)$

$\leq\left(1 / C_{j 0}\right)\left(1+\phi_{0} E_{\mu_{0}} / \Phi\right)^{k}$

$\cdot\left[I_{\text {sat }}\left(e^{\alpha \phi_{0} E \mu_{0}}-1\right)+\left(W_{0}+J_{0}\right) /(2 \sqrt{L})\right]$

$. E_{\mu_{0}} e^{(\mu-(R / L))\left(t-t_{k}\right)} /(\mu-(R / L)) \leq \phi_{0} e^{(\mu-(R / L))\left(t-t_{k}\right)}$.

Therefore $B$ maps $M_{W} \times M_{J} \times M_{0}$ into itself.

It remains to show that $B$ is contractive operator. Indeed, for $t \in\left[t_{k}, t_{k+1}\right],(k=0,1,2, \ldots)$ we obtain
$\left|B_{W}\left(W, J, u_{C_{0}}\right)(t)-B_{W}\left(\bar{W}, \bar{J}, \bar{u}_{C_{0}}\right)(t)\right|$

$\leq\left|Z_{0}-R_{0}\right||J(t-T)-\bar{J}(t-T)| /\left(Z_{0}+R_{0}\right)$

$\leq\left|Z_{0}-R_{0}\right||J(t-T)-\bar{J}(t-T)| e^{-\mu\left(t-T-t_{k}\right)} e^{\mu\left(t-T-t_{k}\right)} /\left(Z_{0}+R_{0}\right)$

$\leq\left|Z_{0}-R_{0}\right| \rho_{s}(J, \bar{J}) e^{\mu\left(t-t_{k}\right)} /\left(Z_{0}+R_{0}\right)$.

It follows

$\rho_{k}\left(B_{W}\left(W, J, u_{C_{0}}\right), B_{W}\left(\bar{W}, \bar{J}, \bar{u}_{C_{0}}\right)\right) \leq\left|Z_{0}-R_{0}\right| \rho_{s}(J, \bar{J}) /\left(Z_{0}+R_{0}\right)$.

In a similar way

$\left|B_{J}\left(W, J, u_{C_{0}}\right)(t)-B_{J}\left(\bar{W}, \bar{J}, \bar{u}_{C_{0}}\right)(t)\right|$

$\leq\left(2 \sqrt{L}\left|u_{C_{0}}(t)-\bar{u}_{C_{0}}(t)\right|+\left|Z_{0}-R_{2}\right||W(t-T)-\bar{W}(t-T)|\right) /\left(Z_{0}+R_{2}\right)$

$\leq\left(2 \sqrt{L} \rho_{k}\left(u_{C_{0}}, \bar{u}_{C_{0}}\right)+\left|Z_{0}-R_{2}\right| \rho_{s}(W, \bar{W})\right) e^{\mu\left(t-t_{k}\right)} /\left(Z_{0}+R_{2}\right)$.

It follows

$\rho_{k}\left(B_{J}\left(W, J, u_{C_{0}}\right), B_{J}\left(\bar{W}, \bar{J}, \bar{u}_{C_{0}}\right)\right)$

$\leq\left(2 \sqrt{L} \rho_{k}\left(u_{C_{0}}, \bar{u}_{C_{0}}\right)+\left|Z_{0}-R_{2}\right| \rho_{s}(W, \bar{W})\right) /\left(Z_{0}+R_{2}\right)$

$\leq\left(2 \sqrt{L}+\left|Z_{0}-R_{2}\right|\right) \max \left\{\rho_{k}\left(u_{C_{0}}, \bar{u}_{C_{0}}\right), \rho_{s}(W, \bar{W})\right\} /\left(Z_{0}+R_{2}\right)$.

Finally for $0<\gamma<1$ we have

$\left|B_{0}^{(k)}\left(V, I, u_{C_{0}}\right)(t)-B_{0}^{(k)}\left(\bar{V}, \bar{I}, \bar{u}_{C_{0}}\right)(t)\right|$

$\leq \int_{t_{k}}^{t}\left|U_{k}\left(V, I, u_{C_{0}}\right)(s)-U_{k}\left(\bar{V}, \bar{I}, \bar{u}_{C_{0}}\right)(s)\right| d s$

$+\left|\frac{t-t_{k}}{t_{k+1}-t_{k}}\right|\left|\int_{t_{k}}^{t_{k+1}} U_{k}\left(V, I, u_{C_{0}}\right)(s) d s-\int_{t_{k}}^{t_{k+1}} U_{k}\left(\bar{V}, \bar{I}, \bar{u}_{C_{0}}\right)(s) d s\right|$

$\equiv W_{1}+W_{2}$.

Since

$W_{1} \leq \int_{t_{k}}^{t}\left|\left(1-\left(u_{C_{0}}(s) / \Phi\right)\right)^{\gamma}-\left(1-\left(\bar{u}_{C_{0}}(s) / \Phi\right)\right)^{\gamma}\right| e^{(\mu-(R / L))\left(s-t_{k}\right)}$

.$\left|I_{s a t}\left(e^{\alpha \phi_{0} E \mu_{0}}-1\right)+\left(W_{0} e^{-\mu T}+J_{0}\right) /(2 \sqrt{L})\right| d s$

$+\left(1 / C_{j 0}\right)\left(1+\left(\phi_{0} E_{\mu_{0}} / \Phi\right)\right) \int_{t_{k}}^{t} e^{(\mu-(R / L))\left(s-t_{k}\right)} d s$

.$\left(\alpha I_{s a t} e^{\alpha \phi_{0} E_{\mu_{0}}} \rho_{k}\left(u_{C_{0}}, \bar{u}_{C_{0}}\right)+\left(\rho_{s}(W, \bar{W})+\rho_{k}(J, \bar{J})\right) /(2 \sqrt{L})\right)$

$\leq\left(\gamma / C_{j 0}\right)\left(1-\left(\phi_{0} E_{\mu_{0}} / \Phi\right)\right)^{\gamma-1}$

$\left(I_{\text {sat }}\left(e^{\alpha \phi_{0} E_{\mu_{0}}}-1\right)+\left(W_{0}+J_{0}\right) /(2 \sqrt{L})\right)$

$\cdot \int_{t_{k}}^{t}\left|\left(u_{C_{0}}(s)-\bar{u}_{C_{0}}(s)\right) / \Phi\right| e^{(\mu-(R / L))\left(s-t_{k}\right)} d s$

$+\left(1 / C_{j 0}\right)\left(1+\left(\phi_{0} E_{\mu_{0}} / \Phi\right)\right) \int_{t_{k}}^{t} e^{(\mu-(R / L))\left(s-t_{k}\right)} d s$

.$\left(I_{s a t}\left(e^{\alpha \phi_{0} E_{\mu_{0}}} \rho_{k}\left(u_{C_{0}}, \bar{u}_{C_{0}}\right)+\left(\rho_{s}(W, \bar{W})+\rho_{k}(J, \bar{J})\right) /(2 \sqrt{L})\right)\right.$

$\leq\left(I_{\text {sat }}\left(e^{\alpha \phi_{0} E_{\mu_{0}}}-1\right)+\left(W_{0} e^{-\mu T}+J_{0}\right) /(2 \sqrt{L})\right)$

.$\left(\gamma / C_{j 0}\right)\left(1-\left(\phi_{0} E_{\mu_{0}} / \Phi\right)\right)^{-1} \rho_{k}\left(u_{C_{0}}, \bar{u}_{C_{0}}\right) \int_{t_{k}}^{t} e^{2(\mu-(R / L))\left(s-t_{k}\right)} d s$

$+\left(1 / C_{j 0}\right)\left(1+\left(\phi_{0} E_{\mu_{0}} / \Phi\right)\right) \int_{t_{k}}^{t} e^{(\mu-(R / L))\left(s-t_{k}\right)} d s$

$\cdot\left(I_{s a t}\left(e^{\alpha \phi_{0} E_{\mu_{0}}} \rho_{k}\left(u_{C_{0}}, \bar{u}_{C_{0}}\right)+\left(\rho_{s}(W, \bar{W})+\rho_{k}(J, \bar{J})\right) /(2 \sqrt{L})\right)\right.$

$\leq\left(\gamma /\left(C_{j 0} \Phi\right)\right)\left(I_{s a t}\left(e^{\alpha \phi_{0} E_{\mu}}-1\right)+\left(W_{0} e^{-\mu T}+J_{0}\right) /(2 \sqrt{L})\right)$ 
. $\left(1-\left(\phi_{0} E_{\mu_{0}} / \Phi\right)\right)^{\gamma-1} \rho_{k}\left(u_{C_{0}}, \bar{u}_{C_{0}}\right)\left(e^{2(\mu-(R / L))\left(t-t_{k}\right)}-1\right) /(2 \mu)$

$+\left(1 /\left(C_{j 0}\right)\left(1+\left(\phi_{0} E_{\mu_{0}} / \Phi\right)\right) \gamma\left(e^{(\mu-(R / L))\left(t-t_{k}\right)}-1\right) / \mu\right.$

$\left(I_{s a t} e^{\alpha \phi_{0} E_{\mu_{0}}} \rho_{k}\left(u_{C_{0}}, \bar{u}_{C_{0}}\right)+\left(\rho_{s}(W, \bar{W})+\rho_{k}(J, \bar{J})\right) /(2 \sqrt{L})\right)$

$\leq 1 /\left(\mu C_{j 0}\right) e^{(\mu-(R / L))\left(t-t_{k}\right)}$

$\cdot\left\{\left(I_{s a t}\left(e^{\alpha \phi_{0} E \mu_{0}}-1\right)+\left(W_{0}+J_{0}\right) /(2 \sqrt{L})\right)\right.$

. $(\gamma / \Phi)\left(1-\left(\phi_{0} E_{\mu_{0}} / \Phi\right)\right)^{-1}\left(E_{\mu_{0}}-1\right) / 2$

$\left.+\left(1+\left(\phi_{0} E_{\mu_{0}} / \Phi\right)\right) r\left[I_{s a t} e^{\alpha \phi_{0} E_{\mu_{0}}}+(1 / \sqrt{L})\right]\right\}$

. $\max \left\{\rho_{k}\left(u_{C_{0}}, \bar{u}_{C_{0}}\right), \rho_{s}(W, \bar{W}), \rho_{k}(J, \bar{J})\right\}$

and

$W_{2} \leq\left(1 /\left(\mu C_{j 0}\right)\right)\left(E_{\mu_{0}}-1\right)$

$\cdot\left\{\left(I_{s a t}\left(e^{\alpha \phi_{0} E_{\mu}}-1\right)+\left(W_{0}+J_{0}\right) /(2 \sqrt{L})\right)(\gamma / \Phi)\right.$

$. e^{(\mu-(R / L))\left(t-t_{k}\right)}\left(1-\left(\phi_{0} E_{\mu_{0}} / \Phi\right)\right)^{-1}\left(E_{\mu_{0}}-1\right) / 2$

$\left.\left.+\left(1+\left(\phi_{0} E_{\mu_{0}} / \Phi\right)\right)\right\rangle\left[I_{s a t} e^{\alpha \phi_{0} E_{\mu_{0}}}+(1 / \sqrt{L})\right]\right\}$

. $\max \left\{\rho_{k}\left(u_{C_{0}}, \bar{u}_{C_{0}}\right), \rho_{s}(W, \bar{W}), \rho_{k}(J, \bar{J})\right\}$

then

$\left|B_{0}^{(k)}\left(W, J, u_{C_{0}}\right)(t)-B_{0}^{(k)}\left(\bar{W}, \bar{J}, \bar{u}_{C_{0}}\right)(t)\right|$

$\leq e^{(\mu-(\mu-(R / L)))\left(t-t_{k}\right)} E_{\mu_{0}} /\left(\mu C_{j 0}\right)$

$\cdot\left\{\left(I_{\text {sat }}\left(e^{\alpha \phi_{0} E_{\mu_{0}}}-1\right)+\left(W_{0}+J_{0}\right) /(2 \sqrt{L})\right)(\gamma / \Phi)\right.$

. $\left(1-\left(\phi_{0} E_{\mu_{0}} / \Phi\right)\right)^{-1}\left(E_{\mu_{0}}-1\right) / 2$

$\left.\left.+\left(1+\left(\phi_{0} E_{\mu_{0}} / \Phi\right)\right)\right\rangle\left[I_{s a t} e^{\alpha \phi_{0} E_{\mu_{0}}}+(1 / \sqrt{L})\right]\right\}$

. $\max \left\{\rho_{k}\left(u_{C_{0}}, \bar{u}_{C_{0}}\right), \rho_{s}(W, \bar{W}), \rho_{k}(J, \bar{J})\right\}$.

Therefore

$\rho_{k}\left(B_{0}^{(k)}\left(W, J, u_{C_{0}}\right), B_{0}^{(k)}\left(\bar{W}, \bar{J}, \bar{u}_{C_{0}}\right)\right)$

$\leq K_{C} \rho_{j(k)}\left(\left(W, J, u_{C_{0}}\right),\left(\bar{W}, \bar{J}, \bar{u}_{C_{0}}\right)\right)$

where

$K_{C}=E_{\mu_{0}}\left(1+\phi_{0} E_{\mu_{0}} / \Phi\right)^{\gamma /\left(\mu C_{j 0}\right)(\gamma / \Phi)}$

$\cdot\left\{\left(I_{s a t} e^{\alpha \phi_{0} E_{\mu_{0}}}+\left(W_{0}+J_{0}\right) /(2 \sqrt{L})\right)\left(1-\phi_{0} E_{\mu_{0}} / \Phi\right)^{-1}\left(E_{\mu_{0}}-1\right) / 2\right.$

$\left.+I_{s a t} e^{\alpha \phi_{0} E_{\mu}}+(1 / \sqrt{L})\right\}$.

Finally we obtain

$\rho_{k}\left(\left(B_{W}\left(W, J, u_{C_{0}}\right), B_{J}\left(W, J, u_{C_{0}}\right), B_{0}\left(W, J, u_{C_{0}}\right)\right)\right.$,

$\left.\left(B_{W}\left(\bar{W}, \bar{J}, \bar{u}_{C_{0}}\right), B_{J}\left(\bar{W}, \bar{J}, \bar{u}_{C_{0}}\right), B_{0}\left(\bar{W}, \bar{J}, \bar{u}_{C_{0}}\right)\right)\right)$

$\leq L_{0} \rho_{j(k)}\left(\left(W, J, u_{C_{0}}\right),\left(\bar{W}, \bar{J}, \bar{u}_{C_{0}}\right)\right)$

where $L_{0}=\max \left\{K_{W}, K_{J}, K_{C}\right\}<1$.

Therefore the fixed point of operator $B$ is an oscillatory solution of the problem (9).

Theorem 1 is thus proved.

\section{NUMERICAL EXAMPLE}

Here we rewrite all inequalities from the main theorem guaranteeing existence-uniqueness of oscillatory solution:

$\left(2 \sqrt{C} Z_{0} W_{0}+\left|Z_{0}-R_{0}\right| J_{0}\right) /\left(Z_{0}+R_{0}\right) \leq W_{0} ;$

$\left(2 \sqrt{L} \phi_{0}+\left|Z_{0}-R_{2}\right| W_{0}\right) /\left(Z_{0}+R_{2}\right) \leq J_{0} ;$

$\left.\left(1 / C_{j 0}\right)\left(1+\phi_{0} E_{\mu_{0}} / \Phi\right)\right)^{y}$

$\cdot\left[I_{\text {sat }}\left(e^{\alpha \phi_{0} E_{\mu_{0}}}-1\right)+\left(W_{0}+J_{0}\right) /(2 \sqrt{L})\right]$

. $E_{\mu_{0}} /(\mu-(R / L)) \leq \phi_{0}$

$K_{W}=\left|Z_{0}-R_{0}\right| /\left(Z_{0}+R_{0}\right)<1 ;$

$K_{J}=\left(2 \sqrt{L}+Z_{0}-R_{2}\right) /\left(Z_{0}+R_{2}\right)<1$;

$K_{C}=E_{\mu_{0}} /\left(\mu C_{j 0}\right)$

$\cdot\left\{\left(I_{s a t} e^{\alpha \phi_{0} E_{\mu}}+\left(W_{0}+J_{0}\right) /(2 \sqrt{L})\right)(\gamma / \Phi)\right.$

. $\left(1-\left(\phi_{0} E_{\mu_{0}} / \Phi\right)\right)^{-1}\left(E_{\mu_{0}}-1\right) / 2$

$\left.+\left(1+\left(\phi_{0} E_{\mu_{0}} / \Phi\right)\right) \gamma\left[I_{s a t} e^{\alpha \phi_{0} E_{\mu_{0}}}+(1 / \sqrt{L})\right]\right\}<1$.

Let us consider a transmission line with length $\Lambda=10 \mathrm{~m}$. The inductance, capacitance and resistance per-unit length are $L=0,45 \mu \mathrm{H} / \mathrm{m} ; C=80 \mathrm{pF} / \mathrm{m} ; R=0,01 \Omega / \mathrm{m} ; R / L=$ $=2,2.10^{4} ; R_{0}=R_{2}=35 \Omega$. Then in order to satisfy the Heaviside condition we have to choose $G=L C / R=0,36.10^{-12} \mathrm{mho} / m ; T=\Lambda \sqrt{L C}=6.10^{-8} ;$ $Z_{0}=\sqrt{L / C}=75 \Omega$. Here $\alpha=q /(\eta K T)$, where $q=1,6.10^{-19} C$ is the electron charge, $K=1,23.10^{-23}$ is the Boltzmann constant; $\quad T=293 \mathrm{~K}\left(20^{\circ} \mathrm{C}\right) \quad$ is absolute temperature. We choose the ideality factor $\eta \in[1 ; 1,2]$ (cf. [2]). The diffusion potential is $\Phi=0,5 \mathrm{~V}, C_{j 0}=5.10^{-11} \mathrm{~F}$ is the zero-voltage junction capacitance, $I_{\text {sat }}=10^{-8} \mathrm{~A}$, $\alpha=37$. If the junction is uniformly doped, $\gamma=0,5$. For $T_{0}=10^{-16}$ we choose $\mu=10^{14} \Rightarrow(\mu-(R / L)) T_{0} \approx 10^{-2}$, $E_{\mu_{0}}=e^{\left(\mu-(R / L) T_{0}\right.}=e^{0,01} \approx 1$ and we take $\phi_{0}=0,16$. Then $\alpha \phi_{0}=37.0,16=5,92 \Rightarrow e^{5,92} \approx 372,5$ and we choose $W_{0}=J_{0}=10^{-4}$. The above inequalities become $2 \sqrt{80.10^{-12}} \frac{75}{75+15}=2.10^{-6} \sqrt{80} \frac{75}{75+15} \leq 1$; $2 \sqrt{0,45 \cdot 10^{-6}} \frac{0,16}{75+15} \leq 10^{-4}$;

$\frac{0,5(1,32)^{1,5}}{10^{14} \cdot 1,5 \cdot 5 \cdot 10^{-11}}\left(10^{-8}(373)+\frac{10^{-4}}{2 \sqrt{0,45 \cdot 10^{-6}}}\right) \leq 0,16 ;$

$K_{W}=\frac{75-35}{75+35}=0,36<1 ; \quad K_{J}=0,36<1 ; L_{0}=0,36 ;$

$K_{C}=\left(1 / \mu C_{j 0}\right)\left(1+\left(\phi_{0} / \Phi\right)\right)^{\gamma}\left(I_{\text {sat }} \exp \left(\alpha \phi_{0}\right)+(1 / \sqrt{L})\right) \approx 0,34$. 


\section{CONCLUSION}

We have noticed that the transmission lines loaded by Schottky diode circuit requires a specific way of derivation of the boundary conditions and respectively leads to different type of differential equations, namely, one could not exclude some transitional currents and so we have to solve a system of three equations for three unknown functions. We have shown that the right-hand side of the differential equations are not periodic functions that implies to look for oscillatory solutions. Introducing a suitable operator whose fixed point are oscillatory solutions we obtain existence-uniqueness of an oscillatory solution. It can be reached by successive approximations as in [8] from the operator equations

$$
\begin{aligned}
& W^{(p)}(t)=\left(2 e^{R t / L} E(t) \cdot 10^{-3} \sqrt{0,45}+40 J^{(p-1)}(t-T)\right) / 110, \\
& J^{(p)}(t)= \\
& =\left(-2 e^{R t / L} u_{C_{0}}{ }^{(p-1)}(t) \cdot 10^{-3} \sqrt{0,45}+40 W^{(p-1)}(t-T)\right) / 110 \\
& u_{C_{0}}{ }^{(p)}(t)=B_{0}\left(W^{(p-1)}, J^{(p-1)}, u_{C_{0}}{ }^{(p-1)}\right)
\end{aligned}
$$

beginning with suitable $W^{(0)}(t), J^{(0)}(t), u_{C_{0}}{ }^{(0)}$. The rate of convergence is

$$
\begin{aligned}
& \rho_{k}\left(\left(W^{(p+1)}, J^{(p+1)}, u_{C_{0}}{ }^{(p+1)}\right),\left(W^{(p)}, J^{(p)}, u_{C_{0}}{ }^{(p)}\right)\right) \leq \\
& \leq \frac{(0,36)^{p}}{1-0,36} \rho_{k}\left(\left(W^{(1)}, J^{(1)}, u_{C_{0}}{ }^{(1)}\right),\left(W^{(0)}, J^{(0)}, u_{C_{0}}{ }^{(0)}\right)\right) .
\end{aligned}
$$

\section{ACKNOWLEDGMENT}

The paper is granted by contract 158/2018, Faculty of Mining and Electro-mechanics, University of Mining and Geology “St. I. Rilski”, 1700 Sofia.

\section{REFERENCES}

[1] W. Schottky, "Zur Halbleitertheorie der sperrschicht - und spitzengleichrichter,“ Zeitschrift fuer Physik, vol. 113, no. 5-6, pp. 367-414, 1939.

[2] S. A. Maas, Nonlinear Microwave and RF Circuits, 2nd ed. Boston London, Artech House, 2003.

[3] V. Damgov, Nonlinear and Parametric Phenomena: Theory and Applications in and Radiophysical Mechanical Systems, World Scientific: New Jersey, London, Singapore, 2004.

[4] D. K. Misra, Radio-Frequency and Microwave Communication Circuits. Analysis and Design, 2nd ed. University of WisconsinMilwaukee, John Wiley \& Sons, Inc., Publication, 2004.

[5] V. G. Angelov, "Oscillations in lossy transmission lines terminated by in series connected nonlinear RCL-loads," Int. J. Theoretical and Mathematical Physics, vol. 2, no. 5, pp. 143-162, 2012.

[6] V. G. Angelov, "Lossless transmission lines terminated by in series connected $R L$-loads parallel to $C$-load," Int. J. Theoretical and Mathematical Physics. vol. 3, no. 1, pp. 10-25, 2013.

[7] V. G. Angelov, "Lossless transmission lines terminated by $L$-load in series connected to parallel Connected GL-loads," British Journal of Mathematics \& Computer Science, vol. 3, no. 3, pp. 352-389, 2013.A.

[8] V. G. Angelov, A Method for Analysis of Transmission Lines Terminated by Nonlinear Loads, New York, Nova Science, 2014.

[9] A. Scott, Active and Nonlinear Wave Propagation in Electronics, New York, Wiley Interscience, J. Wiley and Sons, 1970.

[10] Ishimaru, Electromagnetic Wave Propagation, Radiation and Scattering, New Jersey, Prentice-Hall, Inc., 1991.
[11] P. C. Magnusson, G. C. Alexander, and V. K. Tripathi, Transmission Lines and Wave Propagation, 3rd ed., CRC Press. Boka Raton, 1992.

[12] C. R. Paul, Analysis of Multi-Conductor Transmission Lines, New York, Wiley-Interscience Publication, J. Wiley \&Sons, 1994.

[13] S. Ramo, J. R., Whinnery, and T. van Duzer, Fields and Waves in Communication Electronics, New York, John Wiley \& Sons, Inc. 1994.

[14] Rosenstark S., Transmission Lines in Computer Engineering, New York, Mc Grow-Hill. 1994.

[15] J. Dunlop and D. G. Smith, Telecommunications Engineering, U.K.: Chapman \& Hall, London, 1994.

[16] P. Vizmuller, RF Design Guide Systems, Circuits and Equations, Artech House, Inc., Boston London, 1995.

[17] D. Pozar, Microwave Engineering, New York, J. Wiley \&Sons, 1998.

[18] G. Miano, and A. Maffucci, Transmission Lines and Lumped Circuits, New York, Academic Press, 2001

[19] F. Martín, Artificial Transmission Lines for RF and Microwave Applications, Wiley Series in Microwave \& Optical Engineering. 2015.

[20] R. Singh, Circuit Theory and Transmission Lines, McGraw Hill Education, 2013

[21] V. Makwana and B. Bhalja, Transmission Line Protection Using Digital Technology (Energy System in Electrical Engineering), Springer, 2016.

[22] R. A. Lundquist, Transmission Line Construction: Methods and Costs, Forgotten Books, 2015

[23] Dr. S. Ruikar, Electromagnetics and Transmission Lines, Nirali Prakashan, 2016.

[24] S. Kalaga and P. Yenumula, Design of Electrical Transmission Lines: Structures and Foundations, CRC Press, 2016.

[25] V. G. Angelov, Fixed Points in Uniform Spaces and Applications, Romania, Cluj University Press, Babes-Bolyai University, 2009.

[26] L. P. Georgiev, "Some extension of Angelov's fixed point theorem in uniform space" (to appear).

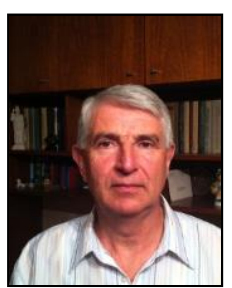

Vasil G. Angelov was born in Sofia, Bulgaria in 1950. He graduated at the Faculty of Mathematics and Mechanics at Sofia University in 1974, received his $\mathrm{PhD}$ in Delay differential equations at Centre of Applied mathematics at Technical University - Sofia in 1981 and DSc in the field of transmission lines at Military Academy - Sofia in 2004.

He was in army in 1974-1975. His work experience started in 1976 as Mathematician, in 1978-1985 he was Assistant Professor, in 1985-2007 - Associate Professor, from 2007 up to now - Professor, Department of Mathematics, Faculty of Mining Electro-mechanics, University of Mining and Geology "St. I. Rilski" - Sofia. He has 126 research papers, 14 text books, and 2 monographs: Fixed Points in Uniform Spaces and Applications, Cluj University Press, Romania, 2009; A Method for Analysis of Transmission Lines Terminated by Nonlinear Loads, New York, Nova Science, 2014. His current and previous research interests are in the field of: Delay Differential Equations, Fixed Point Theory, Classical Electrodynamics, Electrodynamics and Radio-Technical Devices, Transmission Lines Theory, and Nonlinear Circuit Theory.

$\mathrm{He}$ is Member of: the American Mathematical Society, New York Academy of Sciences-1996, European Centre for Research Training and Development, and Expert Council - International Achievements Research Center - Chicago; Member of Editorial Boards of: Bulgarian Institute for Analysis and Investigations, International Journal on Fixed Point Theory, Computation and Applications, International Journal of Theoretical and Mathematical Physics, Journal of Mathematical Sciences and Applications, Results in Nonlinear Analysis, Bulletin of Allahabad Mathematical Society and Reviewer of: Mathematical Reviews, Zentralblatt für Mathemik, Journal „Applied Mathematics E-notes”.

Awards and honors: Who's Who in the World-since 1992; Gold Medal of the American Biographical Institute, Ghost of the World Forum 2010 University of Cambridge, UK; International Peace Prize by the Authority of the United Cultural Convention- USA 2012; Scientist of the Year-2015, Summit of Leaders, Oxford Academic Union, E B A, London. 